\title{
PROPHYLACTIC INTRAMEDULLARY NAILING OF BISPHOSPHONATE RELATED BILATERAL SEQUENTIAL ATYPICAL FEMORAL SHAFT FRACTURE: A TREATMENT STRATEGY
}

Girish H. Rudrappa르, Ye Yeon Won², Vishwanath Yaligod ${ }^{3}$, Umesh M. Shivanna4

\section{HOW TO CITE THIS ARTICLE:}

Girish H. Rudrappa, Ye Yeon Won, Vishwanath Yaligod, Umesh M. Shivanna. "Prophylactic Intramedullary Nailing of Bisphosphonate Related Bilateral Sequential Atypical Femoral Shaft Fracture: A Treatment Strategy". Journal of Evolution of Medical and Dental Sciences 2014; Vol. 3, Issue 51, October 09; Page: 12023-12031, DOI: $10.14260 /$ jemds/2014/3586

ABSTRACT: Even though there are recent reports showing strong association between long term bisphosphonate therapy and atypical femoral shaft fractures, there are no well-defined guidelines regarding screening of these fractures and also regarding prevention of the same in a patient with bilateral sequential atypical femoral shaft fractures. We report our experience with two cases of bilateral, sequential, atypical femoral shaft fractures helpful in formulating guidelines for screening and management of these fractures. We also believe that prophylactic femoral intramedullary nailing with continuation of bisphosphonate therapy as a potential option for preventing the propagation of incomplete bilateral simultaneous/sequential atypical femoral shaft fractures into complete fracture.

KEY WORDS: Bisphosphonate, atypical, fracture, prophylactic, Nailing.

INTRODUCTION: There is growing concern regarding atypical fractures of the femoral diaphysis associated with long term bisphosphonate therapy.1-2 Reports of sequential bilateral atypical femoral shaft fractures have been reported and there are no definite guidelines for screening and management of unaffected side. ${ }^{3,5-7}$ We report two cases of sequential, bilateral, atypical femoral shaft fractures associated with long term bisphosphonate therapy. We review the current literature and discuss not only about screening of unaffected side but also its management and describe the option of prophylactic closed intramedullary nailing of contralateral side as a potential management strategy.

\section{CASE REPORT:}

Case No 1: A 65 year old woman with a spine T-Score of -3.22 on bone densitometry was diagnosed with osteoporosis and treatment started with Alendronate $10 \mathrm{mg}$ per day. After 5 years, patient had a spontaneous onset of pain in the right thigh. Radiograph of right femur revealed an incomplete fracture on tension side of right femoral diaphysis, (Figure1) (a \& b), which was treated conservatively with rest and continuation of alendronate along with calcium and vitamin $\mathrm{D}_{3}$ supplementation. Radiograph of the left femur at this time showed thickening of diaphyseal cortex without an obvious fracture line (Figure 1) (c).

Bone scan revealed focal increased uptake in right mid femoral shaft due to fracture and only a mild uptake in the lateral border of left femur (Figure1) (d). After 18 months, patient developed a complete fracture of right femoral diaphysis which was treated with closed intramedullary nailing (Figure 2) (a \& b), and Alendronate was continued since T score was -2.3. The fracture was mid shaft transverse with beaking and with thick cortices, which resembled a "thumbs up \& thumbs down" pattern (Figure 3). 
This was similar to "simple with thick cortices" pattern described by Lenart BA et al. ${ }^{2}$ The fracture met all the major criteria for atypical femoral fracture as described by 2010 American Society for Bone and Mineral research task force definition ${ }^{8}$. Six months later, patient developed spontaneous onset of pain in the left thigh. Radiograph of left femur revealed an incomplete fracture of the diaphysis on the tension side (Figure 4) (a).

Bone scan revealed increased uptake in the left mid femoral shaft due to fracture and focal intense increased uptake in the right mid femoral shaft due to post traumatic change (Figure 4). Prophylactic closed intramedullary nailing of left femur was done (Figure 4) (c) and Alendronate was continued since $\mathrm{T}$ score was still -2.3. At recent follow-up after 10 yrs of bisphosphonate therapy fractures of both femur were united well (Figure 5) (a \& b) even though T score remained at -2.7.

Case No: 2: Seventy two years old woman was treated with alendronate for osteoporosis for the past 6 years. Within 2 years of starting treatment, she developed pain in her right knee following a trivial fall. Radiograph of the knee was normal but bone scan revealed mild diffuse increased uptake in both patellae and focal intense increased uptake in Right femoral midshaft (Figure 6) (a). Radiographs of both femurs were done which showed an incomplete fracture of the outer cortex of right femoral midshaft with thickening of the cortices (Figure 6) (b). Cortical thickening was also noted in left femur but without fracture (Figure 6) (c). Prophylactic closed femoral nailing of right femur was done (Figure 6) (d) with continuation of alendronate.

Two years late, she developed pain in left thigh after slipping down at home. Radiographs showed an indistinct, incomplete fracture of left femoral midshaft (Figure 7) (a) which was similar to previous fracture of right femur and bone scan showed increased uptake in the left femoral midshaft (Figure 7) (b). Prophylactic femoral nailing was done for left femur also (Figure 7) (c) with continuation of alendronate. At recent follow up, both fractures were well united with no other fractures. (

Figure 8) (a \& b). Metabolic bone diseases other than osteoporosis were ruled out for both patients, and there was no past history of long term medications like steroids which could have been possible confounding factors. ${ }^{3}$

Serum electrophoresis showed no 'M band'. Serum calcium, serum phosphorus, alkaline phosphatase, Vit-D \& parathormone levels were within normal limits. N-terminal telopeptide, a biochemical marker of bone resorption was also within normal limits. Consent was obtained from both the patients after informing that data concerning the case would be submitted for publication.

DISCUSSION: Atypical femoral diaphyseal fractures associated with prolonged bisphosphonate therapy have been described radiologically to demonstrate a simple transverse pattern with hypertrophy of the fractured diaphyseal cortex ${ }^{1,2}$ with thickening of the lateral cortical margin of the unfractured contralateral femur. Our first patient had a midshaft transverse fracture of right femur with beaking and cortices thickened, which resembled a "thumbs up \& thumbs down" pattern (Figure 3). This was similar to "simple with thick cortices" pattern described by Lenart BA et al.2

Recently a register-based national cohort study by Abrahamsen $\mathrm{B}$ et al $^{9}$ found that the ratio between hip and subtrochanteric/diaphyseal fratures was identical in alendronate treated patients and the control cohort even in the limited number of patients who received long-term treatment. 
They concluded that subtrochanteric/diaphyseal femur fractures share the epidemiology and treatment response of classical hip fractures and are best classified as osteoporotic fractures.

However, register-based study has its own limitations and for legal reasons, it was not possible for the authors to review radiographs or patient notes to confirm the diagnosis/fracture pattern. Moreover, the fracture in our patient met all the major criteria for atypical femoral fracture as described by 2010 American Society for Bone and Mineral research task force definition. ${ }^{8}$

Our first patient was on alendronate for 6 years before she developed a complete fracture of right femur with identical radiological findings. Our second patient developed thickening of femoral cortices with incomplete fracture of lateral cortex of right femur as early as 2 yrs after starting bisphosphonate therapy, which prompted us to treat the patient with prophylactic femoral nailing to prevent her from developing complete fracture in future. The same was repeated on the left side.

Bisphosphonates mainly acts by inhibiting the osteoclasts, suppress normal bone turnover and delay or inhibit fracture healing as suggested by Odvina et al ${ }^{10}$ in a small group of patients who sustained low trauma fractures while on long term alendronate. Increased accumulation of microdamage or micro fractures with increased propensity to fracture has been described with bisphosphonate therapy. ${ }^{11}$

Predicting and preventing fracture of contralateral femur is definitely helpful in preventing morbidity. Careful observation is required for contra-lateral femurs due to high incidence of bilateral atypical femoral fractures among patients treated with bisphosphonates for more than 3 years duration. ${ }^{12}$ Various screening modalities which have been described in the literature include magnetic resonance imaging (MRI), isotope bone scanning, micro-computed tomography, positron emission tomography and possibly bone turnover markers. . $^{5-7,13-15}$

$\mathrm{M} \mathrm{H}$ Edwards et $\mathrm{al}^{3}$ highlights the inadequacy of plain radiography and isotope scans in predicting subsequent fracture. In contrast our patients showed a clear increased radioisotope uptake at the site of incomplete fracture (Figure 1) (d), (Figure 4) (b), (Figure 6)(a) \& (Figure 7)(b), and careful examination of plain radiographs showed an indistinct incomplete fracture on the tension side of left femoral diaphysis (Figure 1) (a\&b), (Figure 6) (a), (Figure 6)(b) \& (Figure 7)(a). Eventhough there is no general consensus, we believe that regular screening of contralateral side with plain radiographs and isotope bone scan is beneficial in preventing the catastrophe.

Our first patient was treated with prophylactic closed intramedullary nailing of the contralateral (left) side upon identifying an incomplete fracture on the tension side of femoral diaphysis on plain radiograph and increased uptake on isotope bone scan. The time interval between complete fracture of right femoral diaphysis and identification of an incomplete fracture on contralateral femoral diaphysis was 6 months. So, it is prudent to screen the contralateral femur at least once within 6 months of first fracture or as soon as the patient develops symptom like pain as in the patient we described. Alendronate was continued since the patient still had a subnormal T score on Bone densitometry.

Some authors have advised prophylactic fixation of the contralateral side 3,16 but whether to stop/continue bisphosphonate therapy or to substitute with parathyroid hormone (PTH) analogue is still controversial. Dell R M et al ${ }^{17}$ suggested that stopping bisphosphonate treatment decreases the risk of having a second atypical femur fracture. But there are recent reports which suggest that in addition to stopping bisphosphonates, treating with recombinant paratharmone Teriparatide improves bone quality and healing of atypical femoral fractures. ${ }^{18-22}$ 
Although we had no experience with the use of Teripartide, the fractures in both the patients healed successfully with prophylactic femoral intramedullary nailing and continuation of bisphosphonate therapy. Patients were also supplemented with calcium and vitamin $\mathrm{D}_{3}$. Our observation and results were similar to the reports made by Lee P et al. ${ }^{23}$

But our observations are contradictory to the 2010 report of a task force of the American Society for Bone and Mineral Research, by Shane et al ${ }^{8}$ which suggests discontinuation of bisphosphonates for patients with atypical femoral fractures. Even though sub trochanteric fractures and other atypical fractures at sites like pubic ramus, ischium, rib, and sacrum have been associated with bisphosphonate therapy, ${ }^{1,10}$ our patients did not have any other fracture at recent follow up.

There is no proper guideline for timing of prophylactic fixation of contralateral femur. However, we believe that prophylactic fixation should be considered whenever there is increased uptake in isotope bone scan and/or incomplete fracture on the tension side of femoral diaphysis.

Based on this approach our second patient was treated with bilateral prophylactic intramedullary femoral nailing. Interestingly, there was disappearance of focal uptake in right femur at the time of prophylactic intramedullary nailing of left femur. This finding strongly supports the advantage of prophylactic intramedullary nailing in preventing stress concentration at the tension side of femoral shaft, thus preventing a complete fracture in future.

CONCLUSION: By our experience and by reviewing the current literature, we conclude that plain radiographs and isotope bone scans will be sufficient for screening the patients on long term bisphosphonate therapy and screening should be done at least once in 6 months or as soon as the patient develop symptoms. More research is required in order to frame proper guidelines regarding screening methods, screening interval, and potential management strategies to prevent atypical fracture of contralateral femoral diaphysis.

We believe in continuing bisphosphonate therapy if the T score is subnormal at the time of prophylactic fixation, however, further studies are required to decide between stopping bisphophonates and starting treatment with Teriparatide. We also strongly believe that bilateral prophylactic intramedullary nailing is a potential management strategy for prevention of the propagation of these atypical femoral shaft fractures associated with long term bisphosphonate therapy.

\section{REFERENCES:}

1. Shane E, Burr D, Abrahamsen B et al: Atypical subtrochanteric and diaphyseal femoral fractures: Second report of a task force of the American society for bone and mineral research. J Bone Miner Res. 2014; vol 29, No 1; 1-23.

2. Lenart BA, Lorich DG, Lane JM: Atypical fractures of the femoral diaphysis in postmenopausal women taking alendronate. N engl J Med. 2008; 358 (12):'1304-6.

3. Edwards MH, McCrae FC, Young-Min SA: Alendronate-related femoral diaphysis fracture- what should be done to predict and prevent subsequent fracture of the contralateral side? Osteoporos Int. 2010 Apr; 21(4):701-3. Doi: 10.1007/S00198-009-0986-Y, Epub 2009 Jun 27.

4. Goddard MS, Reid KR, Johnston JC et al: Atraumatic bilateral femur fracture in long term bisphosphonate use. Orthopaedics. 2009; 32: 624-627. 
5. Capeci CM, Tejwani NC; Bilateral low-energy simultaneous or sequential femoral fractures in patients on long-term alendronate therapy. J Bone Joint Surg Am. 2009; 91: 2556-61.

6. Lee JK: Bilateral atypical femoral diaphyseal fractures in a patient treated with alendronate sodium. Int J Rheum Dis. 2009; 12: 149-54.

7. Carmak S, Mahiroqullan $\mathrm{M}$, Kekikci $\mathrm{K}$ et al: Bilateral low-energy sequential femoral shaft fractures in patients on long term bisphosphonate therapy. Acta Orthop Traumatol Turc. 2013; 47(3): 162-72.

8. Shane E, Burr D, Ebeling PR et al: Atypical subtrochanteric and diaphyseal femoral fractures: report of a task force of the American society for bone and mineral research. J Bone Miner Res. 2010; 25: 2267-94.

9. Abrahamsen B, Eiken P, Eastell R: Cumulative alendronate dose and the long-term absolute risk of subtrochanteric and diaphyseal femur fractures: a register-based national cohort analysis. J Clin Endocrinol Metab. 2010; 95: 5258-65.

10. Odvina CV, Zerwekh JE, Rao DS et al: Severely suppressed bone turnover: a potential complication of alendronate therapy. J Clin Endocrinol Metab. 2005 Mar; 90 (3): 1294-301.

11. Stepan JJ. Burr DB, Pavo I et al: Low bone mineral density is associated with bone microdamage accumulation in postmenopausal women with osteoporosis. Bone. 2007 sep; 41 (3): 378-85.

12. Kang J S, Won Y Y, Kim J O et al; Atypical femoral fractures after anti-osteoporotic medication; a Korean multicenter study. International Orthopaedics. 2014 June, Vol 38 (6): 1247-1253.

13. Li J, Miller MA, Hutchins GD et al; Imaging bone microdamage in vivo with positron emission tomography. Bone. 2005 Dec; 37 (6): 819-24.

14. Tang SY, Vashishth D: A non-invasive in vitro technique for the three-dimensional quantification of microdamage in trabecular bone. Bone. 2007, vol 40 (5); 1259-64.

15. Wang X, Masse DB, Leng $\mathrm{H}$ et al: Detection of trabecular bone microdamage by micro-computed tomography. J Biomech. 2007, 40 (5); 3397-3403.

16. Oh CW, Oh JK, Park KC. Prophylactic Nailing of Incomplete Atypical Femoral Fractures. The Scientific World Journal, vol. 2013, Article ID 450148, 4 pages, 2013. doi:10.1155/2013/450148.

17. Dell RM, Greene D, Tran D et al: Stopping bisphosphonate treatment decreases the risk of having a second atypical femur fracture. AAOS annual meeting; 2012 Feb 7-11; San Francisco, CA, USA.

18. Gomberg SJ, Wustrack RL, Napoli N et al: Teriparatide, Vitamin D, and Calcium healed bilateral subtrochanteric stress fractures in a postmenopausal woman with a 13-year history of continuous alendronate therapy. J Clin Endocrinol Metab. 2011; 96: 1627-32.

19. Huang HT, Kang L, Huang PJ et al: Successful teriparatide treatment of atypical fracture after long-term use of alendranate without surgical procedure in a postmenopausal woman: a case report. Menopause. 2012; 19: 1360-3.

20. Mastaglia S, Aguilar G, Rossi E. Rapid resolution with teriparatide in delayed healing of atypical fracture associated to long term bisphosphonate use. J Bone Miner Res. 2012; 27 (suppl 1).

21. Cheung AM, Bleankney R, Kahn A et al: Effect of teriparatide on fracture healing in patients with non-displaced incomplete atypical femur fractures. J Bone Miner Res. 2012; 27 (suppl 1).

22. Chiang CY, Zebaze RM, Ghasem-Zadeh A et al: Teriparatide improves bone quality and healing of atypical femoral fractures associated with bisphosphonate therapy. Bone. 2013; 52: 360-5. 


\section{CASE REPORT}

23. Lee P, Van der wall H, Seibel MJ: Looking beyond low bone mineral density: Multiple insufficiency fractures in a woman with post-menopausal osteoporosis on alendronate therapy. J Endocrinol Invest. 2007; 30: 590-597.

Figure 1: (a) Indistinct incomplete fracture (white arrow) of right femur.

(b) Magnified view showing the fracture.

(c) Thickening of left femoral diaphyseal cortex with no fracture.

(d) Anterior \& posterior view of focal increased radioisotope uptake in right femoral midshaft with only a mild uptake in left femur.

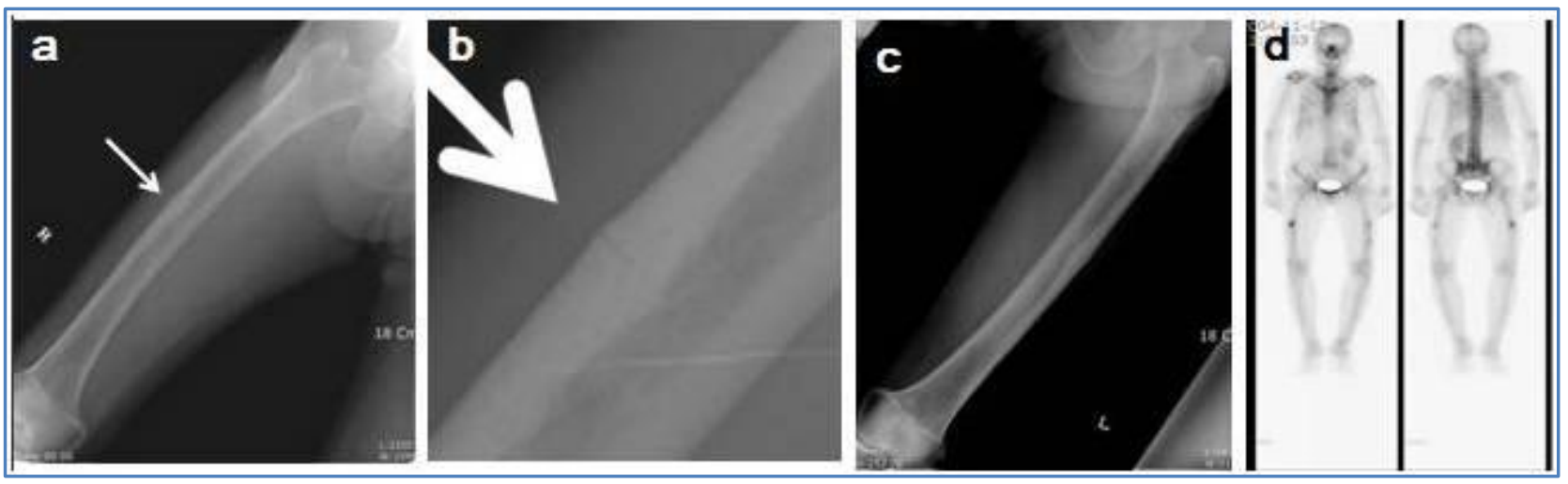

Figure 2: (a) Atypical fracture of right femur showing thick cortices and transverse fracture with beaking (Thumbs up \& Thumbs down pattern).

(b) Closed intramedullary nailing of right femur.

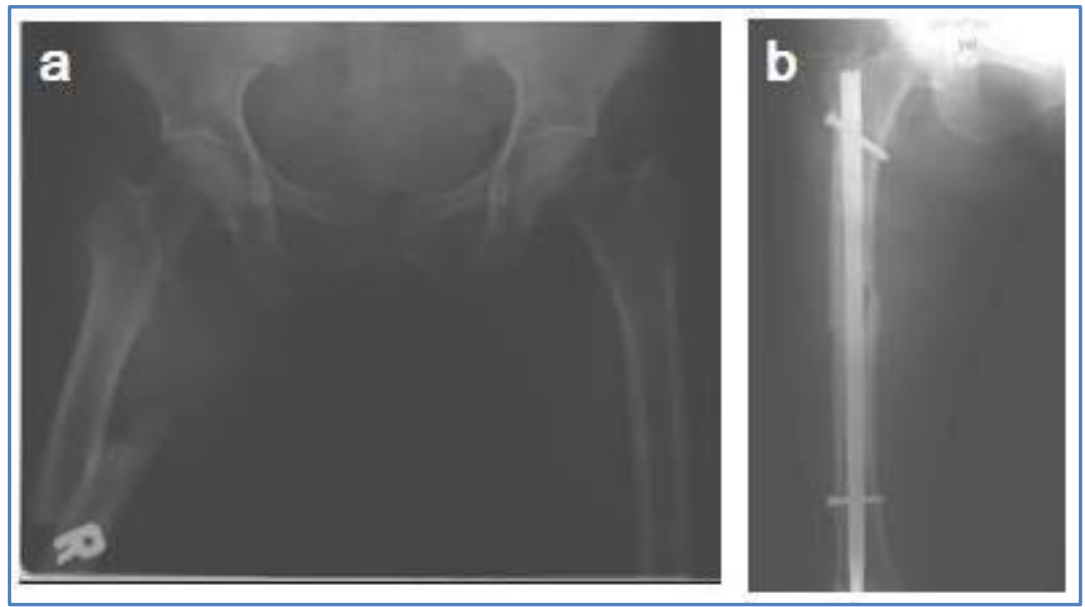




\section{CASE REPORT}

Figure 3: Simplified representation of "thumbs up \& thumbs down" pattern of atypical femoral shaft fracture associated with long term bisphosphonate therapy.

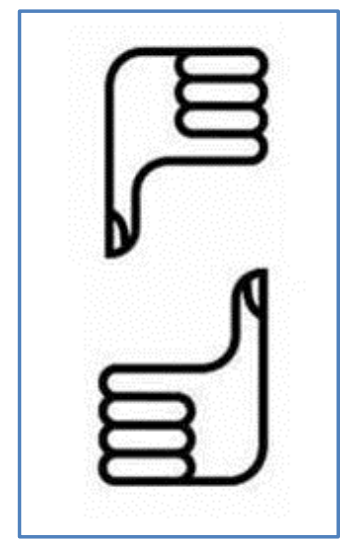

Figure 4: (a) Indistinct incomplete fracture (white arrow) of left femur midshaft.

(b) Increased radioisotope uptake in left femur \& focal intense uptake in right femur due to post traumatic change.

(c) Prophylactic Closed intramedullary nailing of left femur.

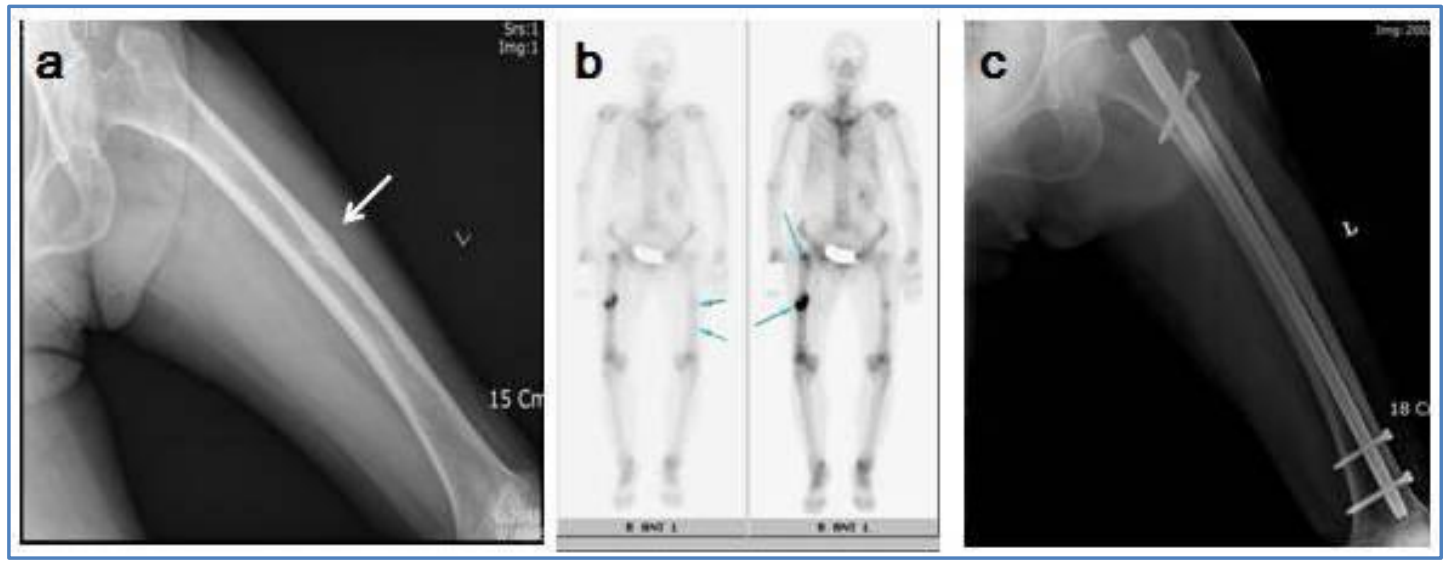

Figure 5: Well united fracture of right femur (a) and left femur (b) at recent follow up.
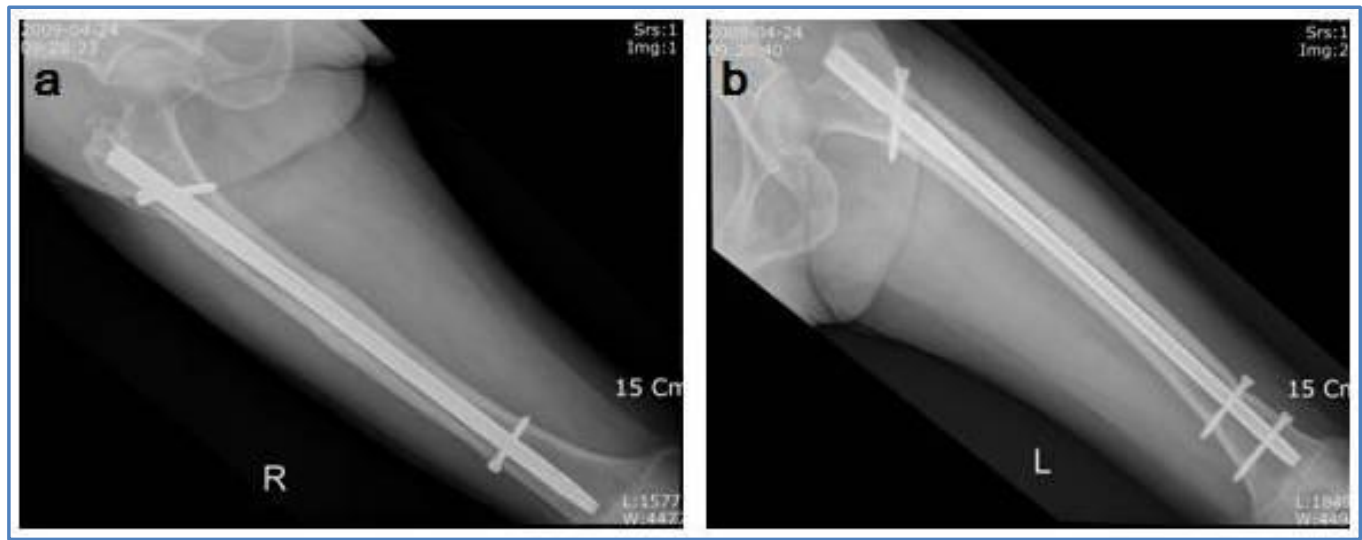


\section{CASE REPORT}

Figure 6: (a) anterior \& posterior view of bone scan showing focal increased radioisotope uptake in right femoral midshaft.

(b) Incomplete fracture (white arrow) of right femoral midshaft.

(c) Cortical thickening of left femoral midshaft without fracture.

(d) Prophylactic femoral nailing of right femur.

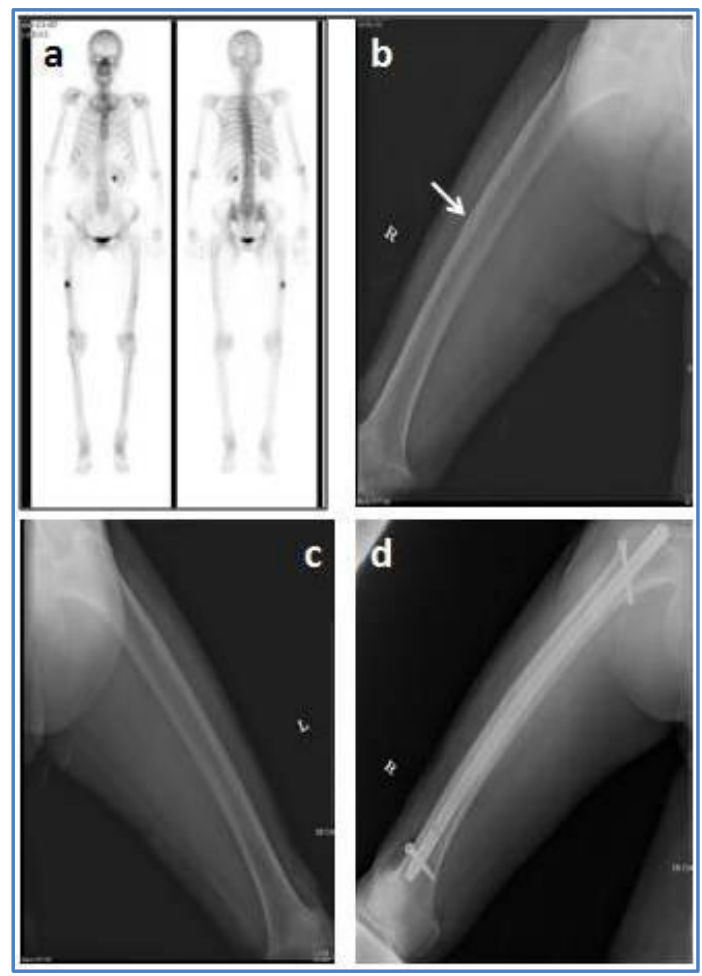

Figure 7: (a) Indistinct incomplete fracture (white arrow) of left femoral midshaft.

(b) Increased radioisotope uptake in left femur \& disappearence of radioisotope uptake in right femur.

(c) Prophylactic intramedullary nailing of left femur.

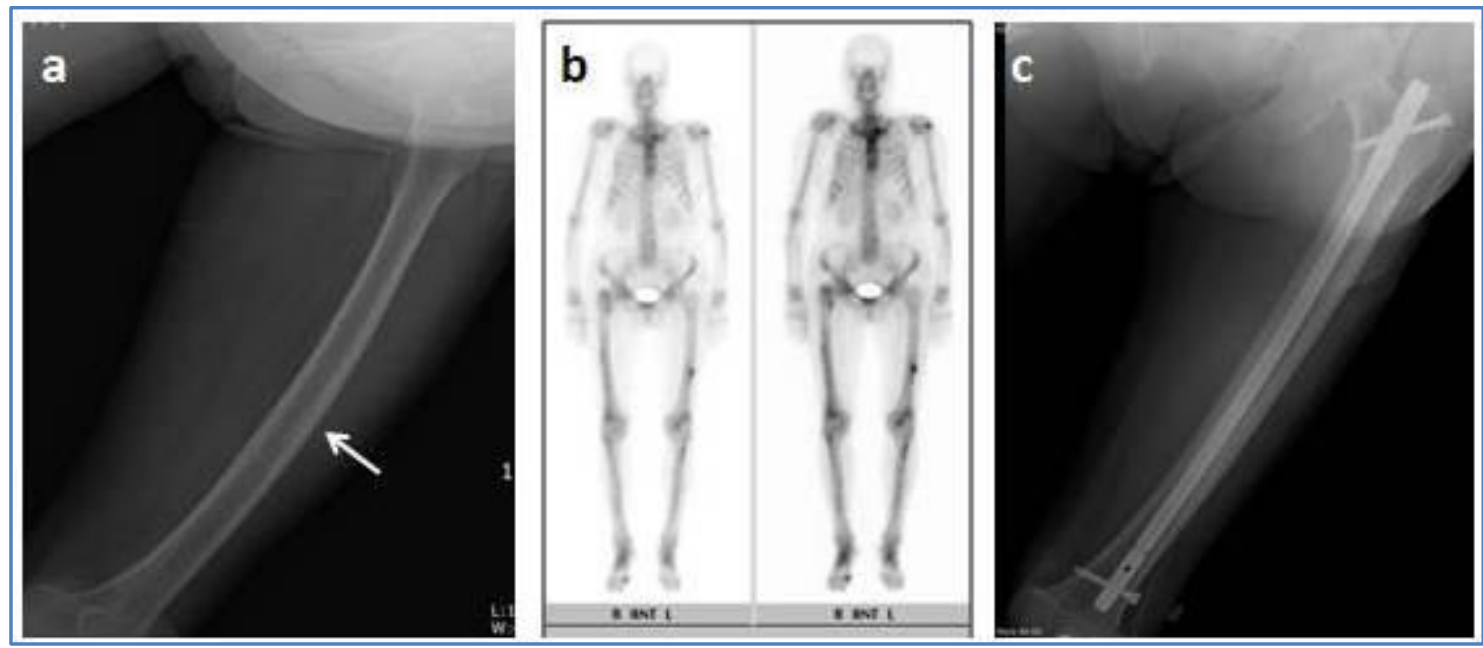




\section{CASE REPORT}

Figure 8: Well united fracture of right femur (a) and left femur (b) at recent follow up.

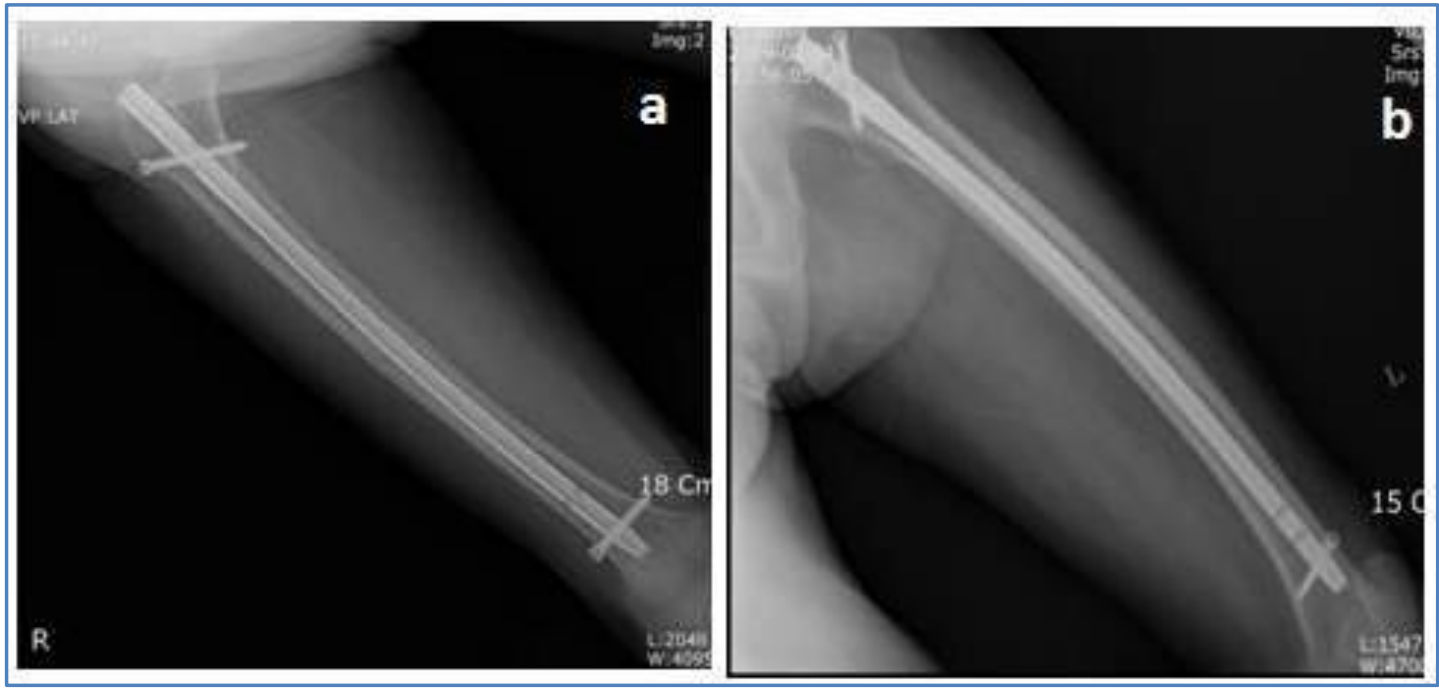

\section{AUTHORS:}

1. Girish H. Rudrappa

2. Ye Yeon Won

3. Vishwanath Yaligod

4. Umesh M. Shivanna

\section{PARTICULARS OF CONTRIBUTORS:}

1. Assistant Professor, Department of Orthopaedics, Sapthagiri Institute of Medical Education \& Research Center, Bangalore.

2. Professor, Department of Orthopaedics, Ajou University School of Medicine, Suwon, South Korea.

3. Professor, Department of Orthopaedics, Sapthagiri Institute of Medical Education \& Research Center, Bangalore.
4. Assistant Professor, Department of Orthopaedics, Sapthagiri Institute of Medical Education \& Research Center, Bangalore.

\section{NAME ADDRESS EMAIL ID OF THE CORRESPONDING AUTHOR:}

Dr. Girish H. Rudrappa, \#978, 15 ${ }^{\text {th }}$ A Cross Road,

Sector A, Yelahanka New Town,

Bangalore-560064.

Email: orthoambition@gmail.com

Date of Submission: 17/09/2014.

Date of Peer Review: 18/09/2014.

Date of Acceptance: 01/10/2014.

Date of Publishing: 09/10/2014. 